\title{
INTEGRAÇÃO DO FRAMEWORK MANAKIN COM A PLATAFORMA DSPACE PARA MÚLTIPLAS APRESENTAÇÕES VISUAIS DE INFORMAÇÕES NOS REPOSITÓRIOS DIGITAIS
}

\author{
José Eduardo Santarem Segundo \\ Liriane Soares de Araújo de Camargo \\ Milton Shintaku \\ Silvana Aparecida Borsetti Gregorio Vidotti
}

\section{Resumo:}

Os repositórios digitais têm sido utilizados atualmente pelas instituições de ensino e pesquisa no Brasil como alternativa para divulgação de seus resultados científicos e acadêmicos, visando principalmente manter a memória e visibilidade institucional. Contudo, a forma de apresentação desses resultados pode influenciar na utilização do mesmo, impactando na interação usuário-sistema por meio dos componentes de interface. Assim, pode-se afirmar que um único ambiente informacional digital pode disponibilizar diversas formas de apresentação visual, customizando componentes informacionais e visuais para comunidades de usuários específicas. Nesse contexto, ferramentas estão sendo desenvolvidas como recurso facilitador ao acesso e uso de informações com o intuito de aumentar a usabilidade de ambientes informacionais digitais. Sendo assim, uma dessas ferramentas é apresentada neste artigo, a qual denomina-se Manakin, bem como sua integração com a plataforma DSpace para possibilitar múltiplas apresentações visuais, visando à importância da diferenciação e direcionamento de interfaces por um mesmo repositório para diversas áreas de conhecimento. Para isso são apresentados resultados e exemplos de repositórios com múltiplas apresentações visuais com o intuito de facilitar a utilização da ferramenta apresentada, bem como reforçar a importância de uma identidade visual diferenciada por área do conhecimento em um mesmo repositório por meio de análise literária e exploratória.

\section{Palavras-chave:}

Dspace; Manakin; Repositórios digitais; Múltiplas apresentações visuais; Interfaces

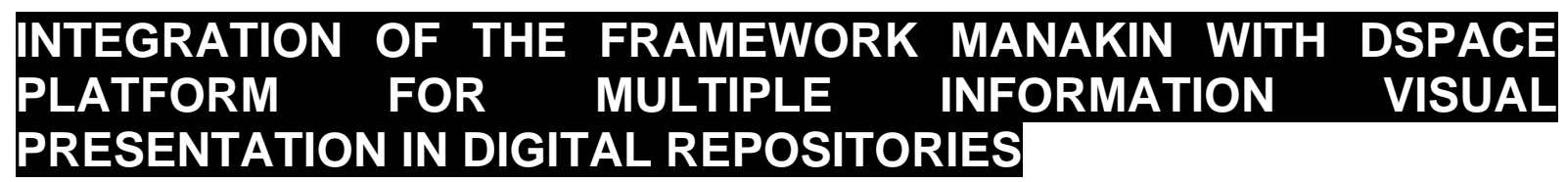

\begin{abstract}
:
Digital repositories are currently being used by education and research institutions in Brazil as an alternative for propagating scientific and academic results, mainly to reach institutional memory and visibility. However, the way these results are presented may influence their use, affecting user-system interaction through the interface components. Thus, it is possible to state that an unique digital information environment can offer different ways of visual presentation, customizing informational and visual components for specific users communities. In this context, tools are being developed as a resource to make access and use of information easier, and to increase the usability of digital informational environments. One of these tools, Manakin, is presented in this paper, as well as its integration with the DSpace platform, in order to enable multiple visual presentations, stressing the importance of the differentiation and direction of interfaces by a single repository to the various knowledge fields. So, results and examples of repositories with multiple visual presentations are introduced, to facilitate the use of the presented tool, as well as to reinforce the importance of a differentiated visual identity by areas of knowledge in a single repository, by means of literary and exploratory analysis.
\end{abstract}

\section{Keywords:}

Dspace; Manakin; Digital repositories; Multiple visual presentations; Interfaces

(c) Revista Digital de Biblioteconomia e Ciência da Informação,Campinas, v.7, n. 2, p. 10-26, jan./jun. 2010- ISSN: 1678-765X. 


\section{Introdução}

A dinamicidade da Internet e seu amplo escopo possibilitam aos pesquisadores diversas formas de navegação e recuperação de informação em diversos ambientes informacionais, os quais devem considerar as características e necessidades dos respectivos públicos-alvo. Contudo, ainda existem muitos ambientes que restringem seus usuários e conteúdos por meio de altos preços de acesso informacional, uso de tecnologia de ponta ou falta de uso de tais tecnologias, dificultando a produção e comunicação científica.

Baseado nesse contexto, surgem ambientes e iniciativas que apóiam o acesso irrestrito e compartilhamento de informações públicas como repositórios e bibliotecas digitais, blogs e wikis que geralmente são baseados em iniciativas como o Movimento de Acesso Livre ou Aberto, que é originado da crise dos periódicos científicos, o qual pode impactar de forma significativa na expansão da disseminação dos trabalhos científicos e acadêmicos.

Assim, considerando esse movimento, este artigo enfoca os ambientes informacionais digitais denominados repositórios digitais, especificamente os repositórios institucionais, os quais inserem-se no movimento conhecido por Open Access Initiative, que visa promover o acesso livre e irrestrito à literatura científica e acadêmica, favorecendo o aumento do impacto do trabalho desenvolvido pelos investigadores e instituições, e contribuindo para a reforma do sistema de comunicação científica (RODRIGUES, 2006).

Vale ressaltar que um repositório institucional é a reunião de todos os repositórios temáticos hospedados em uma organização. No caso de uma universidade, cada departamento trata de uma área do conhecimento e, portanto, seu repositório temático será específico no assunto deste departamento. A união de todos os repositórios das diversas unidades de pesquisa comporá o repositório institucional, caracterizando-o como multidisciplinar (CAFÉ et al., 2003).

Sendo assim, pode-se afirmar que geralmente os repositórios institucionais possuem diversas comunidades e coleções, as quais são destinadas a públicos-alvos diferentes. Assim, as formas de apresentação das informações devem ser planejadas cuidadosamente 
para atender as necessidades dos usuários efetivos, considerando suas respectivas preferências visuais.

Nesse âmbito, pode-se afirmar que vários softwares estão sendo utilizados para auxiliar no desenvolvimento de ambientes informacionais digitais, bem como na elaboração de interfaces. Alguns exemplos são: o E-Prints da Universidade de Southampton ${ }^{1}$ e o DSpace desenvolvido pelo consórcio do Massachussets Institute of Technology - $\mathrm{MIT}^{2} \mathrm{e}$ Hewllet Packard - $\mathrm{HP}^{3}$. Os dois softwares são baseados na filosofia do acesso livre, possuindo código aberto. Contudo o DSpace está sendo mais utilizado pelas instituições, possuindo uma comunidade de apoio oferecida pelo IBICT - Instituto Brasileiro de Informação de Ciência e Tecnologia. E por isso, este artigo enfoca este software, juntamente com a integração de uma ferramenta denominada Manakin, que foi desenvolvida pela Texas A\&M University ${ }^{4}$ para auxiliar na elaboração e customização de interfaces de repositórios.

O objetivo de apresentar a integração dessa ferramenta consiste em mostrar o desenvolvimento de diversas formas de apresentação visual de um mesmo ambiente (repositório institucional) para diversos perfis de usuários, visando o aumento da usabilidade dos repositórios digitais.

A metodologia utilizada consiste no levantamento literário sobre repositórios digitais, principalmente os institucionais, a plataforma DSpace e o Framework Manakin, bem como análise de estudo de casos de repositórios que utilizam a integração do Manakin com o DSpace para múltiplas apresentações de informações.

Espera-se auxiliar desenvolvedores no manuseio da ferramenta apresentada, bem como aumentar a usabilidade dos ambientes por meio de várias formas de apresentação da informação de acordo com perfis de usuários ou comunidades de usuários específicas.

1 Disponível em: <http://eprints.soton.ac.uk/>

2 Disponível em: <http://web.mit.edu/>

3 Disponível em: <http://www.hp.com/>

4 Disponível em: < http://www.tamu.edu/>

(c) Revista Digital de Biblioteconomia e Ciência da Informação,Campinas, v.7, n. 2, p. 10-26, jan./jun. 2010- ISSN: 1678-765X. 


\section{Referencial Teórico}

\subsection{Repositórios Digitais e a plataforma DSpace}

Um repositório digital é uma forma de armazenamento de objetos digitais que tem a capacidade de manter e gerenciar material por longos períodos de tempo e prover o acesso apropriado. A utilização dos repositórios foi possibilitada pela queda dos preços no armazenamento, pelo uso de padrões como o protocolo de coleta de metadados da Iniciativa dos Arquivos Abertos (OAI-PMH), e pelos avanços no desenvolvimento dos padrões de metadados que dão suporte ao modelo de comunicação dos arquivos abertos (VIANA, 2007).

Esses repositórios podem ser institucionais ou temáticos. Os repositórios institucionais podem ser comparados a um agrupamento de repositórios temáticos sob a tutela de uma instituição (CAFÉ et al., 2003). Por agrupar documentos digitais provenientes de diversas áreas do conhecimento, os repositórios institucionais, principalmente das universidades, permitem visualizar as pesquisas desenvolvidas pela instituição.

Como as instituições de ensino superior possuem divisões hierárquicas, relativas a diversas áreas do conhecimento, geralmente, ao criarem um repositório institucional, organizam suas comunidades e coleções de forma a refletir a organização da instituição, transformando o repositório em multitemático. Assim, considerando que um repositório institucional possui em um único ambiente informações para diversas comunidades, pode-se verificar que cada comunidade possui um perfil e formas de navegação e busca distintas. O repositório pode oferecer a esses usuários/comunidades mais de uma opção de interface para utilização do sistema.

As diversas formas de disponibilização da informação (considerando os componentes visuais) oferecem aos usuários opções que podem aumentar a usabilidade do repositório. Contudo, implementar várias interfaces é um esforço árduo para o desenvolvedor que já possui várias preocupações como criação de comunidades e coleções, grupos de usuários, políticas de utilização, auto-arquivamento, interoperabilidade de dados etc.

(c) Revista Digital de Biblioteconomia e Ciência da Informação,Campinas, v.7, n. 2, p. 10-26, jan./jun. 2010- ISSN: 1678-765X. 
Sendo assim, algumas ferramentas estão sendo desenvolvidas para auxiliar no processo de desenvolvimento de repositórios digitais e também no desenvolvimento de várias formas de apresentação visual das informações. Um dos softwares mais utilizados para auxiliar o desenvolvimento de repositórios digitais é o DSpace, que está sendo utilizado em 330 repositórios registrados em mais de 40 países, com mais de um milhão e meio de documentos (DSPACE, 2008).

O Dspace é amplamente utilizado para a implementação de repositórios, principalmente os acadêmicos com 78\% dos repositórios. Os países que mais implementam repositórios com DSpace são: Estados Unidos com 82 repositórios, Inglaterra com 48 repositórios, Canadá com 28 repositório e Japão com 21 repositórios. Dentre os países que implementam repositórios com DSpace, destaca-se a Índia, que possui 15 repositórios cadastrados com 12 pertencentes a institutos de pesquisa e apenas 3 pertencentes a universidades. A África do Sul se destaca no continente africano com 7 repositórios, sendo 5 de universidade e 2 de instituto de pesquisa. A Figura 1 apresenta a distribuição dos repositórios pelos países, por continentes e a relação entre repositórios acadêmicos e institucionais.

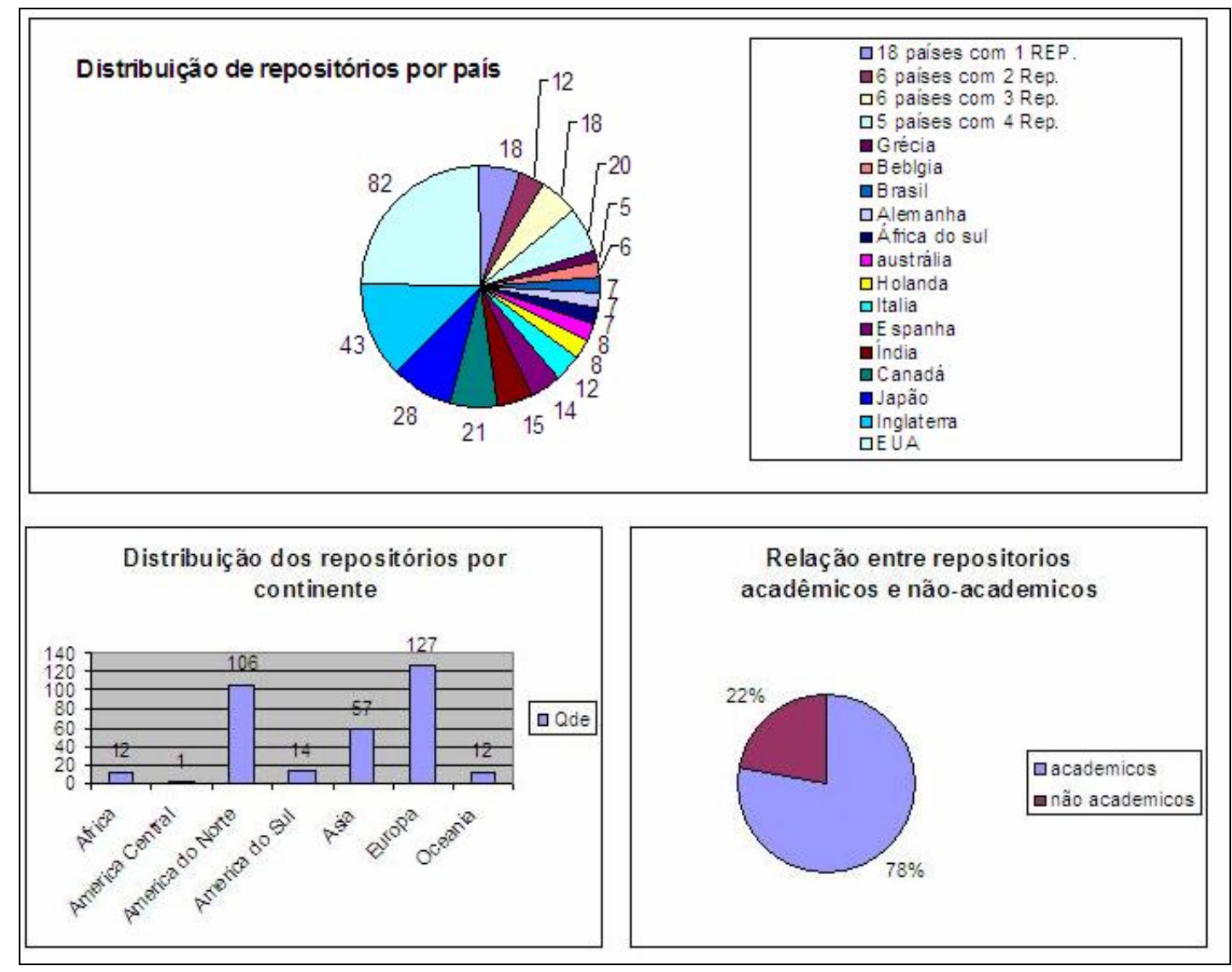

Figura 1: Distribuição do Dspace 
O DSpace (sem alterações locais) permite uma organização hierárquica em comunidades, coleções e itens (metadados e objeto digital). Ele é baseado no padrão Dublin Core de metadados, permitindo o depósito de qualquer tipo de objeto digital (texto, áudio, vídeo etc). O Dspace possui ferramentas de busca simples e avançada, além de possibilitar busca por autor, data, título e assunto e oferece facilidades de alerta sobre novos títulos depositados por meio de e-mail e recurso de sindicação de conteúdo (RSS).

Apesar de basear-se no padrão de metadados Dublin Core - DC, o DSpace permite a implantação de outros esquemas de metadados. Com implantação simples por meio de adequação de alguns arquivos de configuração em formato XML, pode-se implementar os novos campos aos formulários padrão, e incluir novos elementos DC ou novos esquemas. Tal flexibilidade pode facilitar ou dificultar o depósito de vários tipos de documentos científicos, se for considerado que os metadados que descrevem um artigo, geralmente, são diferentes dos metadados que descrevem uma tese, por exemplo.

O DSpace foi desenvolvido no MIT e foi implantado no Brasil primeiramente pela Universidade de São Paulo - USP ${ }^{5}$ e Universidade Federal do Paraná - UFPR ${ }^{6}$, além do Instituto Brasileiro de Informação em Ciência e Tecnologia - IBICT. De caráter inicialmente científico, o DSpace, ganhou outras implementações fora da comunidade científica como a Biblioteca Jurídica - BDJur do Tribunal de Justiça ${ }^{7}$ e a Biblioteca Digital do Senado ${ }^{8}$.

O DSpace apresenta-se atualmente (fevereiro de 2009) na versão 1.5.1 e vem oferecendo várias facilidades a cada nova versão. Da indexação de texto completo oferecida na versão 1.3 às facilidades de uso de vocabulários controlados da versão 1.4. Vale ressaltar que as instituições que implementam repositórios com DSpace desenvolvem facilidades que podem ser agregadas às novas versões do software.

Uma das grandes novidades oferecidas a partir do release 1.5.0 é a agregação das funcionalidades da framework Manakin, que permite formas de apresentação

5 Disponível em: $<$ http://www.usp.br $>$

${ }^{6}$ Disponível em: $<$ http://www.ufpr.br/>

${ }^{7}$ Disponível em: $<$ http://bdjur.stj.gov.br/dspace/>

${ }^{8}$ Disponível em: < http://www2.senado.gov.br/bdsf/ > 
diferenciadas em vários níveis. Essa necessidade é requerida por várias instituições que necessitam de formas de apresentação mais condizentes com a estrutura da instituição e tipos de objetos digitais depositados. Essa ferramenta será detalhada na próxima seção.

\subsection{Framework Manakin}

O Manakin é uma framework para desenvolvimento e customização de interfaces de repositórios, desenvolvido pela Texas A\&M University, que integrado ao DSpace suporta facilidades de customizar as formas de apresentação das informações. É uma extensão que completa de forma harmônica a estrutura do DSpace.

Apresentado com a versão 1.0, no “2 ${ }^{\text {nd }}$ International Conference on Open Repositories”, no ano de 2007 em San Antonio no Texas (EUA), como uma ferramenta independente, para auxiliar na configuração de apresentação dos repositórios que utilizam-se da ferramenta DSpace, ganhou destaque principalmente com a aplicação de suas funcionalidades a alguns repositórios digitais, com destaque para o brasileiro Repositório do Instituto Antonio Carlos Jobim ${ }^{9}$.

O Manakin encontra-se hoje na versão 1.1 e a partir da versão 1.5.0 do DSpace passou a fazer parte do pacote padrão de instalação da ferramenta de repositório, ou seja, na instalação do Dspace 1.5.0, o pacote Manakin é automaticamente instalado. Uma outra nomenclatura utilizada para apresentar o Manakin 1.1 pela Texas A\&M University é como a segunda versão/release do DSpace XML UI.

O framework Manakin apresenta uma série de funcionalidades com destaque para a alteração da apresentação dos dados armazenados e para facilidades em customizar a interface de apresentação de comunidades, coleções e/ou itens dentro de um repositório. A customização da interface é realizada por meio da configuração de temas que passam a ser parte fundamental no desenvolvimento da apresentação visual das informações.

Segundo o Dspace System Documentation (2008, manual da versão 1.5.0 do Dspace),

\footnotetext{
${ }^{9}$ Disponível em: <http://www.tomjobim.org $>$ 
O desenvolvimento de interface com o Manakin é composto de dois principais componentes: aspectos e temas. Os aspectos são como extensões ou plugins para o Manakin; são os componentes interativos que modificam características existentes ou fornecem características novas para o repositório digital. Os temas são os responsáveis por estilizar a apresentação do repositório, da comunidade, ou da coleção.

Os aspectos que definem as características dos repositórios também são responsáveis pelas funcionalidades do repositório, visto que as características acima citadas são exatamente as funções que podem ser utilizadas pelos usuários quando navegam pela ferramenta. Dentro do ambiente fornecido pelo Dspace, o programador responsável pela configuração do ambiente pode criar, excluir ou alterar aspectos utilizando o Manakin, ou ainda dentro de cada aspecto pode inserir ou retirar funcionalidades. É importante ressaltar que as funcionalidades que não fazem parte dos aspectos configurados para determinado repositório não aparecerão para utilização dos usuários, portanto, se, por exemplo, a funcionalidade de mudança de senha for retirada dos aspectos do repositório, os usuários não terão como alterar suas senhas por meio da interface do DSpace.

A configuração original das funcionalidades do Manakin/DSpace estão contidas em quatro aspectos, e podem ser encontradas no manual da versão 1.5.0 do Dspace. Elas são:

- Aspecto Artifact Browser - responsável pelas seguintes funcionalidades: navegação por comunidades, coleções, item e objetos além de visualização de itens individuais e pelas buscas no repositório;

- Aspecto E-Person - responsável pelas seguintes funcionalidades: login e logout do sistema, registro de novos usuários, gerenciamento de senhas esquecidas, gerenciamento de perfis de usuário e alterações de senha;

- Aspecto Submission - responsável pelas seguintes funcionalidades: submissão de novos itens para o repositório, determinação do processo de workflow e postagem de novos itens para o repositório;

- Aspecto Administrative - responsável pelas seguintes funcionalidades: administração do Dspace, como criar, modificar e excluir comunidades, coleções, usuários, grupos e autorizações.

Se os aspectos cuidam das funcionalidades, os temas são os principais responsáveis pela característica de apresentação visual do repositório e podem ser definidos para cada parte 
ou item dentro de um repositório, sendo possível definir um tema padrão para o repositório de uma forma geral e aplicar temas diferentes para uma determinada comunidade, coleção ou item. Desta forma é possível criar um repositório totalmente padronizado ou ainda construir ambientes em que cada comunidade ou coleção tenha apresentações visuais diferenciadas, criando inúmeras possibilidades de desenvolvimento de interfaces que permitam a constituição de um repositório com múltiplas apresentações.

Dentro do ambiente da framework Manakin a indicação de qual tema será utilizado em cada parte ou item do repositório é configurada por meio do arquivo "xmlui.xconf”, que pode ser visto na Figura 2, em que é indicado o tema "Reference” para uma comunidade específica e para todo o restante do repositório, assim como indica também o item “Classic” para uma coleção específica.

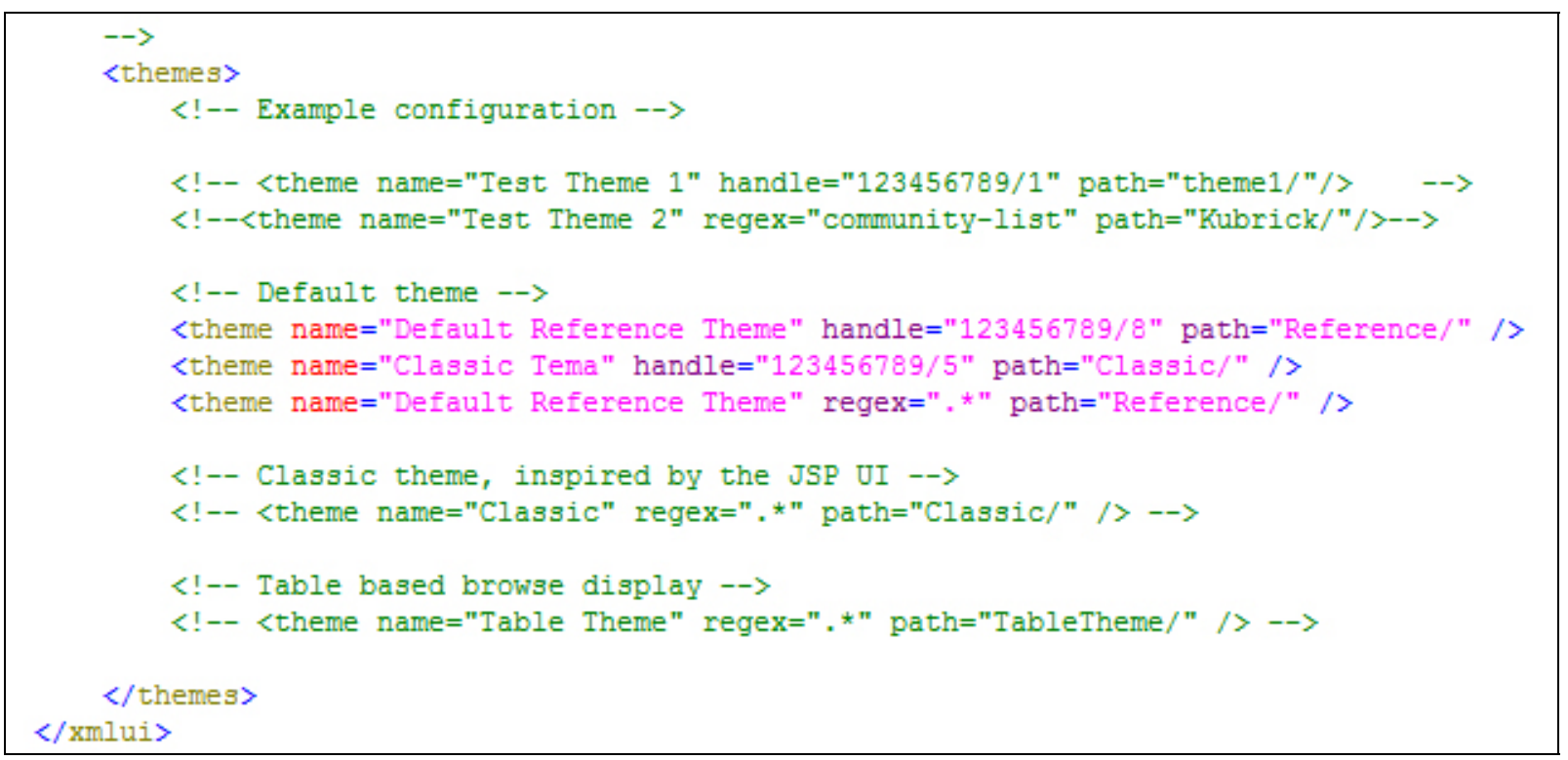

Figura 2: Exemplo de configuração do arquivo "xmlui.xconf”

Além das características de apresentação de interface facilitadas pelo Manakin, vale destacar como uma das principais características da ferramenta a sua capacidade de permitir a divisão estratégica do repositório em camada de negócios e camada de apresentação. Essa característica permite fazer com que sejam realizadas mudanças na camada de apresentação das informações sem a necessidade de fazer alterações na estrutura/arquitetura da informação do repositório. Essa característica vem facilitar e proporcionar as iniciativas para que desenvolvedores possam criar novos módulos e funcionalidades ao Dspace sem a preocupação de ter que alterar as configurações 
principais da ferramenta, evitando prejuízos quando houver futuras atualizações e versão do software.

Uma das vantagens facilmente verificadas pela separação da camada de negócios da camada de apresentação é sua facilidade em criar um repositório multi-idiomas a partir da criação de arquivos que constituem catálogos dos textos utilizados dentro do repositório e que são programados para serem utilizados de acordo com a configuração de língua do browser que o usuário estiver utilizando.

A divisão da camada de negócios da camada de apresentação vem diretamente ao encontro das principais metodologias de desenvolvimento de sistemas para internet que sugerem fortemente padrões de projeto para facilitar a construção e reúso das ferramentas desenvolvidas.

\section{Repositórios Digitais com múltiplas apresentações visuais}

A organização hierárquica do DSpace, com o nível mais alto representado pelas comunidades, se adéqua bem às estruturas das instituições de ensino e pesquisa. Podemse compor as comunidades como aqueles grupos que se inter-relacionam em torno de um tópico, representando as faculdades e institutos de uma universidade ou departamentos de uma instituição de pesquisa.

A idéia de comunidades relacionada com compartilhamento de conceitos (Kuhn, 2006), compõe a estrutura organizacional da maioria das instituições acadêmicas e de pesquisa. Por exemplo, Engenharia Elétrica e Física, mesmo possuindo similaridades ficam em departamentos, faculdades ou institutos diferentes, portanto comporiam comunidades diferentes.

As diferenças disciplinares, geralmente, refletem em muitos aspectos, não somente acadêmico, mas de percepção estética. As páginas das faculdades de um modo geral diferem-se em estrutura e layouts, mesmo pertencendo a uma mesma universidade. A preocupação artística da Música ou Letras, por exemplo, nem sempre é compartilhada com a Química ou Ciência da Computação.

(c) Revista Digital de Biblioteconomia e Ciência da Informação,Campinas, v.7, n. 2, p. 10-26, jan./jun. 2010- ISSN: 1678-765X. 
Pode-se citar como exemplo de uma instituição que apresenta uma diversidade em área do conhecimento a UNESP - Universidade Estadual Paulista ${ }^{10}$, que é constituída por mais de 30 faculdades espalhadas por mais de 20 cidades do estado de São Paulo, e caracteriza-se principalmente pela grande diferença existente entre seus campus. Essa instituição possui uma identidade visual única e padronizada para os sites de todas suas unidades, não diferenciando os diversos institutos, faculdades e departamentos, não aproveitando as diferenças existentes entre tais subdivisões.

Sendo assim, pode-se considerar que a construção de um repositório com múltiplas apresentações deve ser uma das soluções para permitir com que apenas um único ambiente informacional consiga apresentar a produção de uma instituição, identificando os itens de maneira que possam ter uma característica visual que indique a área de conhecimento ou a comunidade em que o conteúdo informacional foi criado.

As duas Figuras (3 e 4) apresentam duas comunidades de um mesmo repositório com identidades visuais diferentes. É importante ressaltar que esta pesquisa não se preocupou em estudar itens de arquitetura da informação ou de aperfeiçoamento de identidade visual para cada uma das comunidades e sim enfocar a utilização de apresentações visuais diferentes, sem tratar da estrutura, cor ou itens de customização.

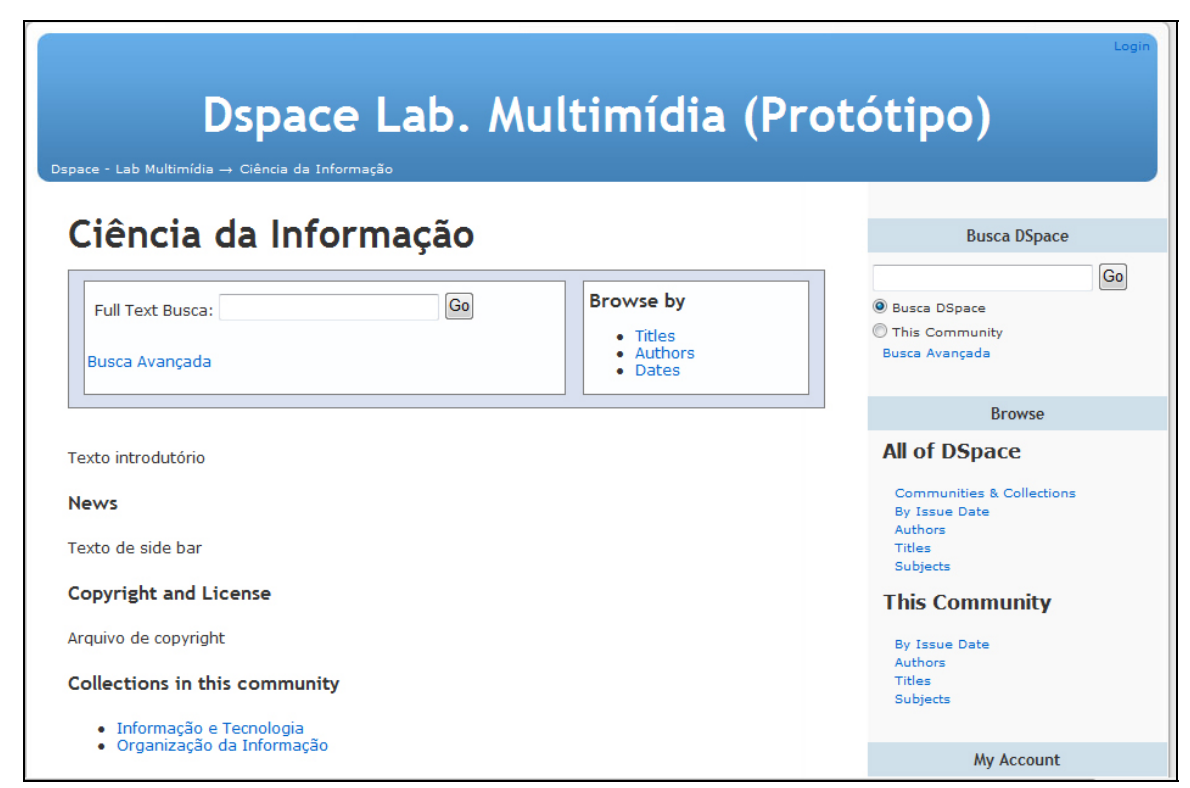

Figura 3: Identidade visual da comunidade “Ciência da Informação” em protótipo de repositório com múltiplas apresentações.

\footnotetext{
${ }^{10}$ Disponível em: <http://www.unesp.br> 


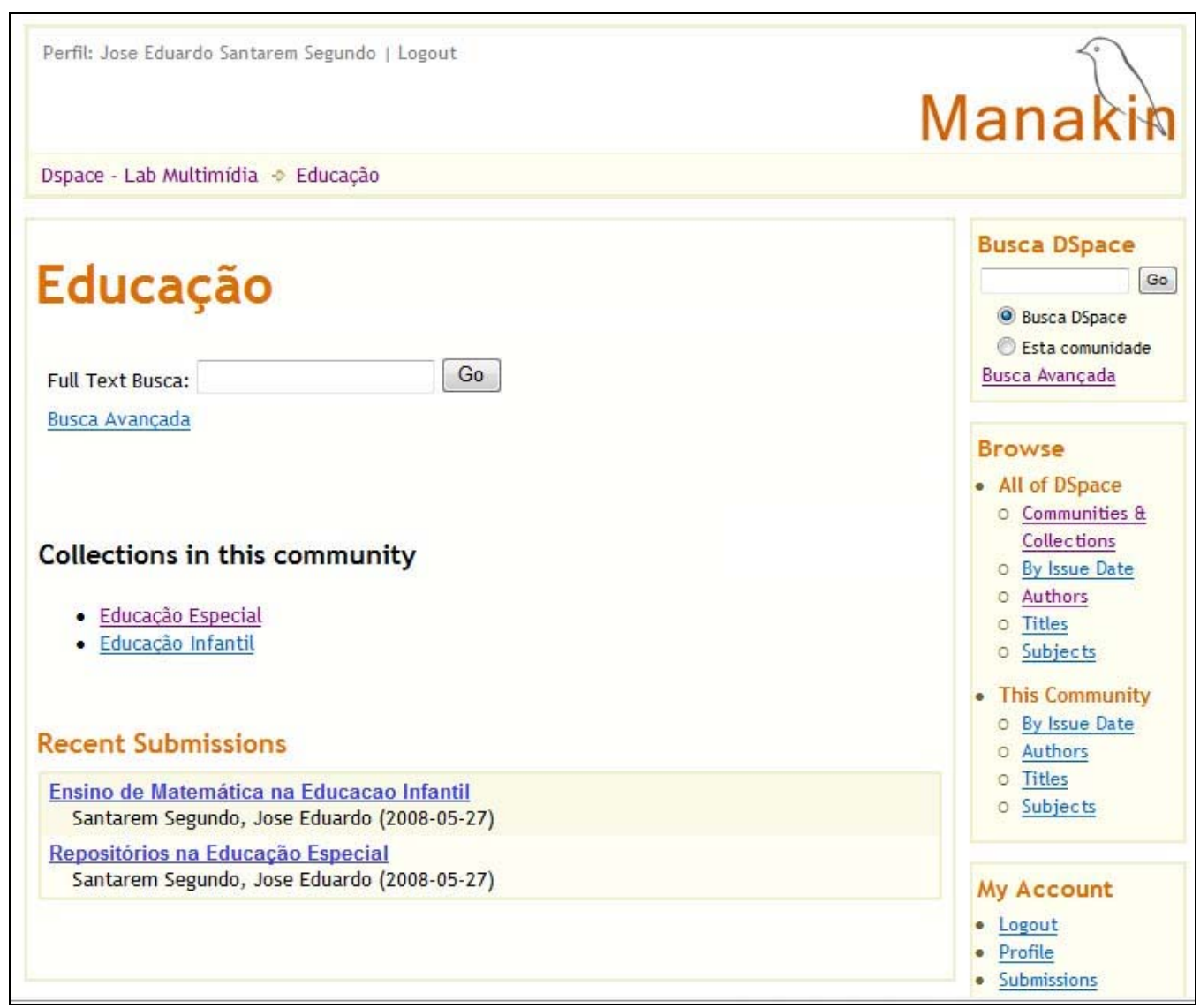

Figura 4: Identidade visual da comunidade "Educação” em protótipo de repositório com múltiplas apresentações.

As duas figuras apresentam interfaces diferentes, porém referem-se ao mesmo repositório. Além das apresentações visuais diferentes para cada uma das comunidades é possível editar e personalizar as coleções, itens ou ainda criar estruturas visuais que identifiquem as informações e registros que estão cadastrados nos repositórios.

A Figura 5 apresenta uma lista resultante de uma pesquisa onde o item é sinalizado com o logo da comunidade a que faz parte, facilitando a identificação da unidade produtora. A Figura 5 foi retirada do protótipo de repositório desenvolvido dentro do Laboratório Multimídia da UNESP de Marília em parceria com o IBICT. É importante ressaltar que esta implementação de lista com identificador em forma de logotipo é uma experiência similar a visualizada na Biblioteca Digital das Universidades do Texas ${ }^{11}$, que é um repositório multiuniversidade que engloba a produção das universidades texanas congregadas ao consórcio. O repositório da Biblioteca, desenvolvido com base no DSpace, está inserido em um projeto amplo, que provê infra-estrutura à várias iniciativas, todas de acesso aberto, como: Repositório, periódico científico, blogs e wikis. 


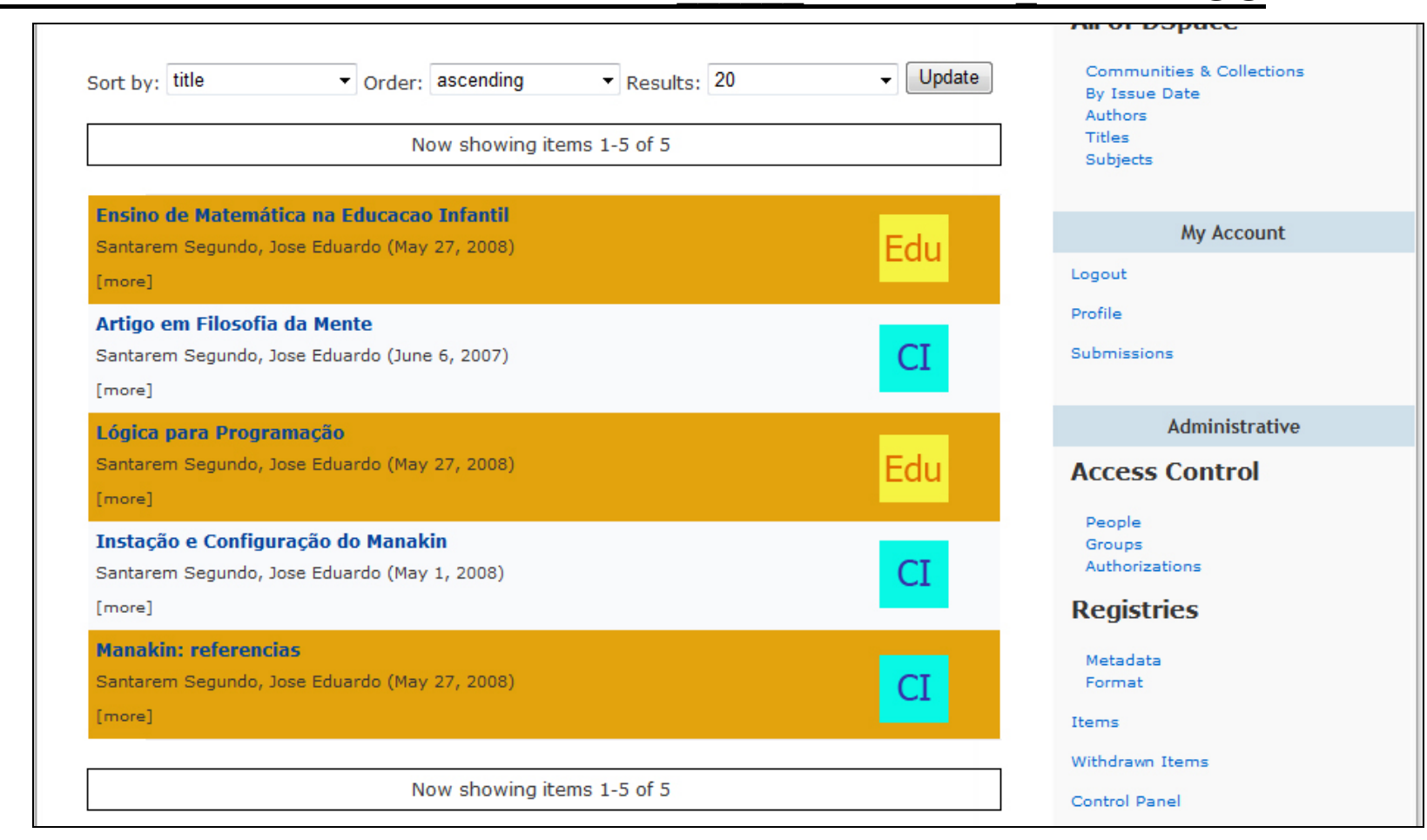

Figura 5: Resultado de busca, com indicação no resultado, através do logotipo, da comunidade originária de onde o documento foi postado.

Na Figura 5 pode-se visualizar itens documentais advindos da área de CI - Ciência da Informação e EDU - Educação em uma interface de recuperação de informação, mostrando aos usuários a origem e localização de cada documento.

A possibilidade de desenvolvimento de ambientes com múltiplas apresentações facilita o processo de adequação do ambiente aos usuários visto que é possível atingir públicos diferentes de acordo com perfis de comunidades.

Apesar deste artigo não se aprofundar no estudo da arquitetura da informação dentro de cada comunidade, é fato que a personalização torna-se assim um processo importante na construção de repositórios com múltiplas apresentações. Camargo e Vidotti (2007) entendem que a personalização é ponto fundamental no desenvolvimento de ambientes digitais, relatando que

A personalização em ambientes de busca pode facilitar e agilizar a pesquisa e melhorar a comunicação entre comunidades virtuais, fazendo com que o usuário se sinta livre para seguir sua própria linha de aquisição de informação para construção do conhecimento no seu próprio ritmo. Além disso, ela pode auxiliar também na distribuição, na

\footnotetext{
${ }^{11}$ Disponível em: <http://www.tdl.org/>

(c) Revista Digital de Biblioteconomia e Ciência da Informação,Campinas, v.7, n. 2, p. 10-26, jan./jun. 2010- ISSN: 1678-765X.
} 
recuperação e na disseminação de informações para diversas comunidades de usuários. Essas comunidades necessitam de uma estrutura amigável para estudar, pesquisar, analisar e manipular informações. A personalização tenta oferecer essa estrutura, atingindo públicos-alvo específicos.

Juntamente com a personalização e a customização deve-se tratar da qualidade, usabilidade e acessibilidade dos ambientes informacionais com o intuito de facilitar o uso do ambiente, visando contribuir com a produção e comunicação científica de forma geral.

\section{Considerações Finais}

Considerando que informações e interfaces direcionadas a comunidades de usuários específicas aumentam e melhoram a utilização de ambientes informacionais digitais, pode-se concluir que a construção de um repositório com múltiplas apresentações visuais permite customizar componentes visuais com o intuito de aumentar a usabilidade do mesmo, bem como colaborar no processo de identificação da origem do documento postado, agilizando e facilitando a recuperação da informação pelo usuário. Nesse contexto, os repositórios multitemáticos que identificam a origem do item em um resultado de busca ajuda a contextualizar a informação, principalmente em caso de busca por termo polissêmico, ou seja, termos que possuem mais de um significado.

Uma ferramenta que está sendo muito utilizada para auxiliar na construção de várias interfaces para um mesmo ambiente é o framework Manakin, que auxilia na construção de interfaces gráficas amigáveis, facilitando o processo de adaptação da interface gerada pela ferramenta Dspace, além de facilitar a implementação de uma arquitetura da informação determinada.

Durante a criação do protótipo de um repositório, utilizando a framework Manakin, foi verificada a necessidade de focar a arquitetura da informação para cada comunidade, visto que antes da utilização do framework, a construção de diversas identidades visuais dentro de um mesmo repositório não era possível.

O uso do Manakin fornece novas possibilidades na construção de novas interfaces visuais e estimula o uso do repositório, tornando o Dspace um ambiente mais agradável e fácil de utilizar, além de estimular o desenvolvimento de novas funcionalidades, permitindo que elas sejam construídas independentemente da estrutura do Dspace.

(c) Revista Digital de Biblioteconomia e Ciência da Informação,Campinas, v.7, n. 2, p. 10-26, jan./jun. 2010- ISSN: 1678-765X. 


\section{ARTIGO}

Com a utilização do framework Manakin pode-se perceber que a utilização e configuração dos aspectos proporcionadas pela camada desse framework facilitam o processo de gerenciamento do repositório pelo administrador, pois possibilita a inserção de funcionalidades de acordo com o público-alvo. E a utilização dos temas facilita a identificação por parte dos usuários quanto à relação do documento selecionado com a área ou departamento em que ele está localizado, criando um novo elemento na tentativa de facilitar o processo de recuperação da informação por parte dos usuários de repositórios digitais.

A tendência é aumentar a utilização do framework Manakin, pois ele cria possibilidades tanto de apresentação visual quanto de configuração de funcionalidades em um repositório digital. Além desses benefícios, pode-se considerar também a crescente utilização de repositórios digitais pelas instituições no mundo.

Assim, o uso e desenvolvimento de novos temas para os repositórios passa a ser uma nova tendência com o uso do framework Manakin, visto as característica de apresentação no formato de camadas sustentados pela tecnologia XML com XSL, o que proporciona a reutilização e também o compartilhamento do código de apresentação entre comunidades que utilizam ou pretendem utilizar repositórios baseados na ferramenta DSPACE.

O uso e configuração dos aspectos vem propiciar aos administradores dos repositórios uma nova característica de administração da ferramenta que é justamente a personalização e adequação do conjunto de funcionalidades de acordo com a instituição ou comunidade de usuários. A configuração dos aspectos permite ajustar o repositório, por exemplo, para que tenha inicialmente funcionalidades básicas, e à medida que o público passe a utilizar e adaptar-se a ferramenta, o administrador responsável pela implementação do repositório vai ter a condição de alterar, liberando ou bloqueando funcionalidades para o repositório de um modo geral, ou de maneira segmentada por áreas divididas por coleções ou comunidades.

Desenvolver novas interfaces visuais para repositórios baseados em DSpace desprendia tempo, considerando o surgimento de futuras versões da ferramenta. Contudo, com a utilização do Manakim essa tarefa se torna mais viável, pois, a separação da camada de apresentação, no que diz respeito à criação de temas, facilita a manutenção e o desenvolvimento de novos temas, principalmente para disponibilizá-los como templates para que outras instituições possam aproveitá-los e reusá-los. 
Sendo assim, pode-se afirmar que a separação da camada de apresentação da camada de negócios vai gerar uma nova perspectiva do uso da ferramenta DSPACE e eliminar algumas limitações relacionadas a sua utilização, mesmo que o desenvolvimento da camada de apresentação esteja sujeita ao trabalho de uma equipe técnica especializada com conhecimentos da tecnologia XML/XSL. Dentro deste contexto é importante ressaltar também a preocupação no desenvolvimento do Manakin com a internacionalização do software DSPACE, já que esse está sendo utilizado de forma crescente.

A ampliação do uso do Dspace resultou na aplicação da internacionalização na camada de apresentação com o uso da ferramenta i18n, acrônimo da palavra internalization, que permite a conversão e adaptação da ferramenta a vários idiomas. Essa conversão é realizada com a utilização de identificadores que são utilizados dentro de programas e convertidos por meio de arquivos que fazem a relação desses identificadores com suas devidas traduções em outras línguas. Dessa forma, é possível que a apresentação do repositório tenha características visuais mais adequadas além da adaptação de língua ao usuário.

Desta forma, a comunidade DSPACE foi contemplada com características importantes na versão 1.5.0, principalmente no que se refere ao aumento de usabilidade da própria ferramenta e na construção e uso de técnicas da arquitetura da informação em um ambiente digital.

\section{Referências}

CAFÉ, L. et al. Repositórios institucionais: uma nova estratégia para publicação científica na Rede. In: ENCONTRO NACIONAL DE INFORMAÇÃO EM CIÊNCIAS DA COMUNICAÇÃO, 13., 2003. Belo Horizonte. Anais... Belo Horizonte, 2003.

CAMARGO, L. S. de A. de; VIDOTTI, S. A. B. G. Personalização: um serviço mediador em ambientes de pesquisa. TransInformação, Campinas, v. 19, n. 3, p. 251-264, set./dez. 2007.

DSPACE System Documentation. Disponível em: < http://www.dspace.org/index.php?option=com_content \&task=view\&id=151\&Itemid=116>. Acesso em: 08 maio 2008.

KUHN, T. S. A estrutura das revoluções científicas. 9. ed. São Paulo: Perspectiva, 2006.

RODRIGUES, E. et al. RepositoriUM: implementação do DSpace em português: lições para o futuro e linhas de investigação. Disponível em: < https://repositorium. sdum.uminho.pt/handle/1822 /679>. Acesso em: 15 maio 2008. 
VIANA, C. L. M.; MÁRDERO ARELLANO, M. Á.; SHINTAKU, M. Repositórios institucionais em ciência e tecnologia: uma experiência de customização do DSpace. In: SIMPÓSIO INTERNACIONAL DE BIBLIOTECAS DIGITAIS, 3., 2005. São Paulo. Proceedings... São Paulo, 2005. p. 1-27. Disponível em

$<$ http://dici.ibict.br/archive/00000719/>. Acesso em: 18 maio 2008.

José Eduardo Santarem Segundo

Doutorando e mestre em Ciência da Informação pela UNESP. santarem@marilia.unesp.br

\section{Liriane Soares de Araújo de Camargo}

Doutoranda e mestre em Ciência da Informação pela UNESP. liriane@marilia.unesp.br

\section{Milton Shintaku}

Mestrando em Ciência da Informação na UnB, pós-graduação Latus Census em Análise de Sistemas pela UnB. miltonshintaku@hotmail.com

\section{Silvana Aparecida Borsetti Gregorio Vidotti}

Doutora em Educação pela UNESP, mestre em Ciência da Computação pela USP - Universidade de São Paulo. vidotti@marilia.unesp.br

Recebido em: 19/03/2009

Aceito para publicação em: jul/2009 